\title{
Delay of Disorder by Diluted Polymers
}

\author{
A.V. Kityk and C. Wagner ${ }^{2, *}$ \\ 1 Institute for Computer Science, Technical University of Czestochowa, \\ Electrical Eng. Dep., Al. Armii Krajowej 17, PL-42200 Czestochowa, Poland \\ 2 Experimentalphysik, Universität des Saarlandes, Postfach 151150, \\ 66041 Saarbrücken, Germany * c.wagner at mx.uni-saarland.de
}

\begin{abstract}
We study the effect of diluted flexible polymers on a disordered capillary wave state. The waves are generated at an interface of a dyed water sugar solution and a low viscous silicon oil. This allows for a quantitative measurement of the spatio-temporal Fourier spectrum. The primary pattern after the first bifurcation from the flat interface are squares. With increasing driving strength we observe a melting of the square pattern. It is replaced by a weak turbulent cascade. The addition of a small amount of polymers to the water layer does not affect the critical acceleration but shifts the disorder transition to higher driving strenghs and the short wave length - high frequency fluctuations are suppressed.
\end{abstract}

It is known from the 1950s [1] that small amounts of flexible polymers can cause drag reduction in turbulent flow. But both experimental and theoretical investigations have not yet conclusively answered the questions what the fundamental mechanisms of drag reduction are and statistical and stability arguments coexist [2]. A difficulty in the theoretical description of drag reduction in fully developed turbulence (e.g. in pipe flow) is that the polymer effect, the drag reduction, occurs in a range where any theoretical treatment of the flow must be fully nonlinear. So called weak turbulence 3], reported for different systems like semiconductor lasers an waves, obeys similar properties like fully developed turbulence, e.g. the energy is injected on large scales and transported via a continuous cascade to shorter, viscous length scales. But typical Reynolds numbers are much lower and for the analytical treatment small background waves can be taken as the small parameter around which a linearisation can be performed.

In this Letter we present experimental measurements that show how polymers can affect the transition to a weakly turbulent state and delay the disordering. We present quantitative data of parametrically driven waves (Faraday waves, see Fig. 1) at the interface of two liquid layers. Due to the low viscosities the system evolves already at low driving strengths from the square state into disorder. Our measurements show that by adding even of a small amount of flexible polymers (Polyethylenoxide, $\mathrm{PEO}$ ) to the aqueous layer the transition into disordered state occurs at considerably higher driving accelerations whereas the critical acceleration of the primary bifurcation remains unaffected. We will see that the pronounced elongational viscosity of the polymer solution accounts for this behavior.

The experimental setup is described in detail elsewhere [4]. In order to overcome the problems associated with light refraction at the interface of a free surface we chose to work with a system of two layers of refractive index matched unmiscible liquids of different densities. The replacement of air as the upper fluid for free surface waves

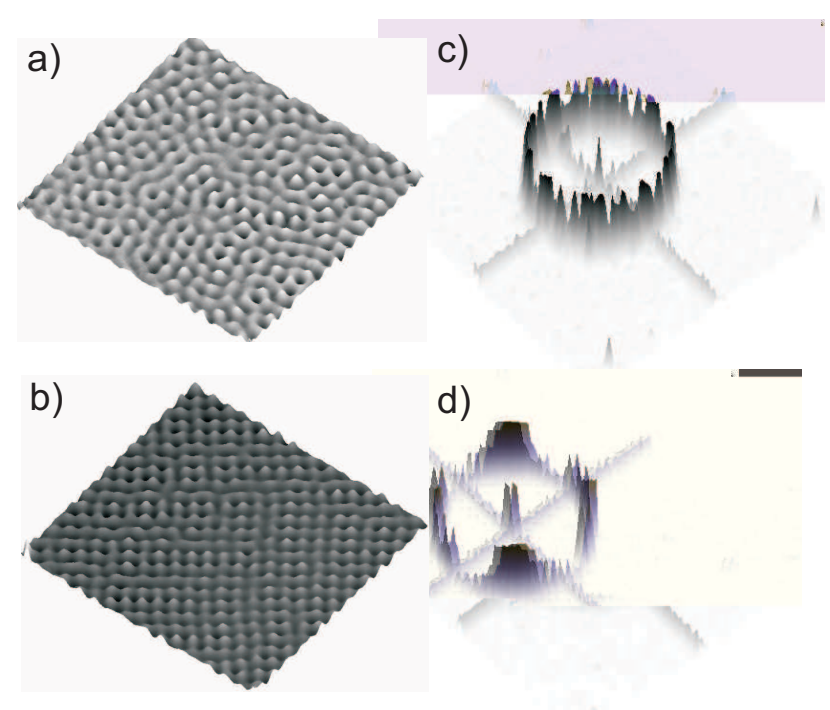

FIG. 1: Snapshots of the interfacial waves at a driving amplitude $a_{0}=31.7 \mathrm{~m} / \mathrm{s}^{2}$. The lower fluid layer is (a) the pure dyed water sugar solution and (b) with 110 ppm PEO. (c) and $(\mathrm{d})$ are the corresponding power spectra.

is hydrodynamically nothing but an increase in viscosity and density, but the actual interfacial deformation can be simply determined by the absorption of light passing the lower, dyed layer. The method has already been proven to allow for quantitative determination of the complete spatio-temporal spectrum of ordered wave states, with a resolution in amplitude better than one percent of the maximum wave height. The lower liquid is a sugardye-water mixture (density $\rho=1200 \mathrm{~kg} / \mathrm{m}^{3}$, viscosity $\eta=3.2 \mathrm{mPas})$ in which the high molecular PEO polymer $\left(M w=6 \times 10^{6} a m u\right)$ can be dissolved. The highest polymer concentration is $110 \mathrm{ppm}$ well below the critical overlap concentration $c^{\star}=300 \mathrm{ppm}$. The upper liquid is a silicon oil (density $\rho=910 \mathrm{~kg} / \mathrm{m}^{3}$, viscosity $\eta=0.65$ $\mathrm{mPas}$ ). The low viscosity was chosen to minimize the influence of the upper layer, similar like in free surface 
waves. The interfacial tension is $\sigma=35$ dynes $/ \mathrm{cm}$, yielding a capillary length of $\ell_{c}=\left(\sigma /(\Delta \rho g)^{1 / 2}=3 \mathrm{~mm}\right.$, with $g$ the earth acceleration and $\Delta \rho$ the differences in the fluid densities. The thickness of the water sugar (silicon oil) layer is $h_{0}=3 \mathrm{~mm}(7 \mathrm{~mm})$. A closed circular vessel with glass plates at the top and bottom is attached to an electromagnetic shaker. The temperature is hold constant at $T=23 \pm 0.1^{\circ} \mathrm{C}$. The system is accelerated in the form $a(t)=a_{0} \sin (\Omega t)$ and the driving frequency is always $\Omega / 2 \pi=47 \mathrm{~Hz}$. We are in the regime of the generic subharmonic response of the Faraday Experiment and the waves oscillate with a fundamental frequency $\omega_{0}=\Omega / 2$. It is related via the dispersion relation to a wavelength $2 \pi / k_{0}=5 \mathrm{~mm}$ and we are close to the infinite depth limit $\tanh \left(k_{0} h\right)=1$. Our measurements are performed by increasing the driving strength $a_{0}$ in steps of $2 \%$. After an equilibration time of 60 seconds the interface is filmed for two seconds by an 8-bit camera with a spatial resolution of $256 \times 256$ pixels at repetition rate of $500 \mathrm{~Hz}$. The maximum acceleration is $a_{0}=95 \mathrm{~m} / \mathrm{s}^{2}$ and for $a_{0}>54 \mathrm{~m} / \mathrm{s}^{2}$ first droplet ejections occur, but only for $a_{0}>70 \mathrm{~m} / \mathrm{s}^{2}$ the interface disintegrates significantly.

All solutions show the same critical acceleration $a_{c}=$ $17.5 \mathrm{~m} / \mathrm{s}^{2}$ at which the interface becomes unstable first. Near onset a pattern of squares is formed. Figure 1 shows a typical snapshot of a measurement at a driving amplitude of $a_{0}=31.7 \mathrm{~m} / \mathrm{s}^{2}$ with the lower layer (a) the water sugar solution and (b) after the addition of 110 ppm PEO. Strikingly, for the diluted polymer solution the quadratic order is still clearly to identify while the order in the water sugar solution is already "melted". A typical temporal realization of the interface displacement $h(x, y, t)$ is shown in Fig. 22 For the polymer solution we observe a small drift while the water sugar solution is strongly intermittent.

The melting processus can be characterized by the normalized angular autocorrelation function $\mathcal{C}\left(\phi^{\circ}\right)$, defined by van de Water and Binks [6] such that square pattern will lead to a pronounced peak at $\mathcal{C}\left(\phi=90^{\circ}\right)$ (Fig. 3). For the polymer solution a quadratic order is found up to driving strength $a_{0} \approx 30 \mathrm{~m} / \mathrm{s}^{2}$. For the water sugar solution the $\mathcal{C}\left(\phi=90^{\circ}\right)$ peak is much weaker and disappears for $a_{0}>20 \mathrm{~m} / \mathrm{s}^{2}$.

Figure 4 shows the temporal and angular averaged amplitudes $\mathbf{F}_{A}$ of the Fourier spectrum. The prominent peak at the basic wave number $k_{0}$ is clearly recognized. This is the length scale at which energy is injected into the system. However, already at intermediate driving strength $a_{0}=31.7 \mathrm{~m} / \mathrm{s}^{2}$ the higher harmonic spatial Fourier peaks are very weak and the spectrum almost consists of a continuum only. The theory for weak turbulent capillary waves [3] predicts a wave number density equivalent to

$$
\mathbf{F}_{A} \sim k^{-15 / 4} .
$$

The exponent could be confirmed by experiments with a

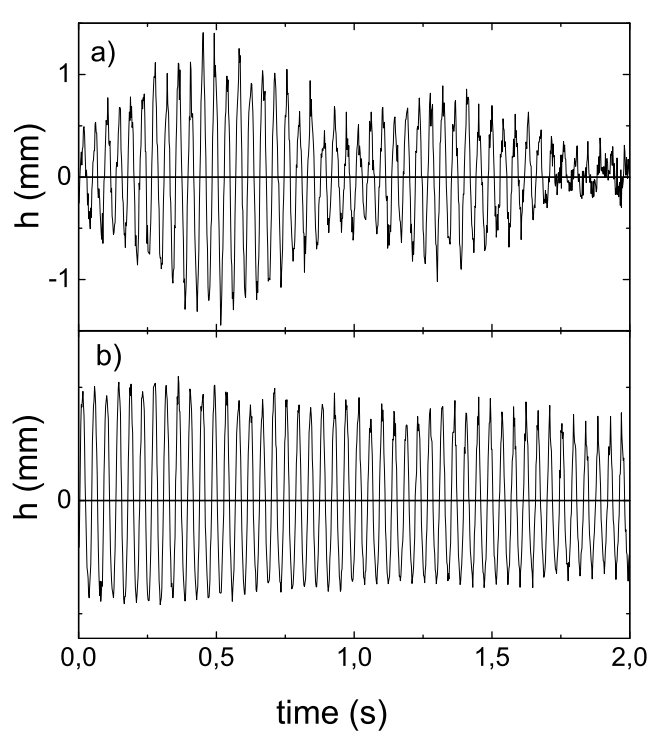

FIG. 2: A typical temporal evolution of the interfacial deformation at an arbitrary point near the center of the container. Parameters are like in Fig. 1]

free surface [5] and our interfacial waves show the same dependency at higher driving strengths, too (see straight line in Fig. (4).

We find a $5 \%$ difference in the basic wave vector $k_{0}$ for the water and the polymer solution but for lower driving strengths the main difference appears at large wave numbers. For the polymer solutions the fluctuations in the small length scales - equivalent to high temporal frequencies [7] - are almost one order of magnitude lower than for the solution without polymers. The integrated intensity $\mathbf{F}_{I}$ (see Fig. 5) is dominated by the power of the basic wave vector and is similar for both systems at $a_{0} \leq 40$ $\mathrm{m} / \mathrm{s}^{2} \mathbf{F}_{I}$. This is in contrast to earlier Faraday Experiments with more viscous polymer solutions [8], where e.g. the critical acceleration is significantly affected due to viscoelasticity. Only for driving accelerations $a_{0} \geq 40$ $\mathrm{m} / \mathrm{s}^{2}$ the integrated intensities $\mathbf{F}_{I}$ of the solution with polymers is lower than for the pure solution (see Fig. 4 and (5).

We think that the phenomena observed in our experiments can be only interpreted if one takes into account the differences in elongational and shear viscosity. A simple, Newtonian liquid is defined by a constant viscosity $\eta$ that does not depend on the applied shear rates or flow forms. Already in linear response the viscosity of viscoelastic liquids becomes a complex quantity $\eta^{*}(\omega)=\eta^{\prime}(\omega)+i \eta^{\prime \prime}(\omega)$, with $\eta^{\prime}$ the dissipation and $\eta^{\prime \prime}$ the elasticity. It can be measured as e.g. in an oscillatory shear experiment with small shear amplitudes. In a similar experiment with constant shear rate $\dot{\gamma}$ polymer solutions might show a shear thinning behavior and $\eta(\dot{\gamma})$ 


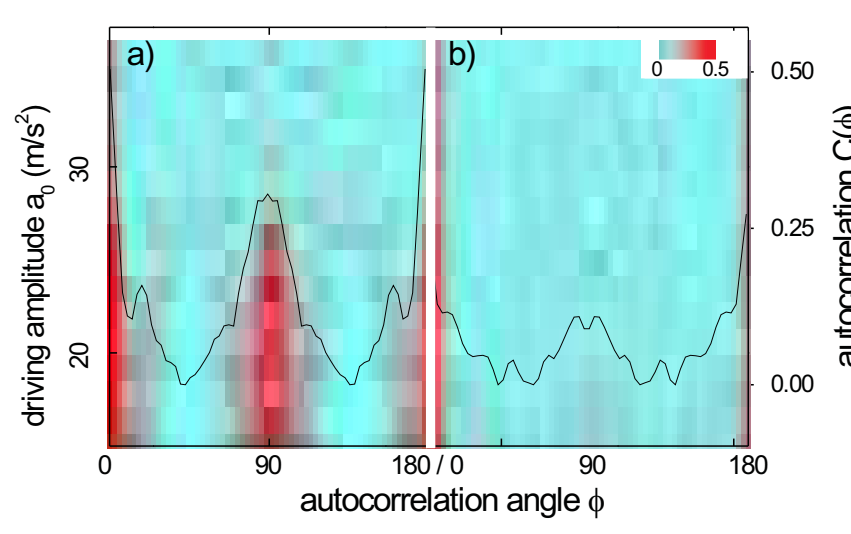

FIG. 3: (Color online:) The temporal averaged and baseline subtracted angular autocorrelation functions $\mathcal{C}\left(\phi^{\circ}\right)$ of the Power spectrum of the interfacial deformations. The color density plot shows the evolution of the autocorrelation function versus driving strength (left y-axes). The quadratic order in the water sugar solution is weak and a standard plot of the autocorrelation function at $a_{0}=25 \mathrm{~m} / \mathrm{s}^{2}$ is shown as an example (black line, right y-axes). The lower fluid layer is (a) the pure dyed water sugar solution and (b) with $110 \mathrm{ppm}$ PEO.

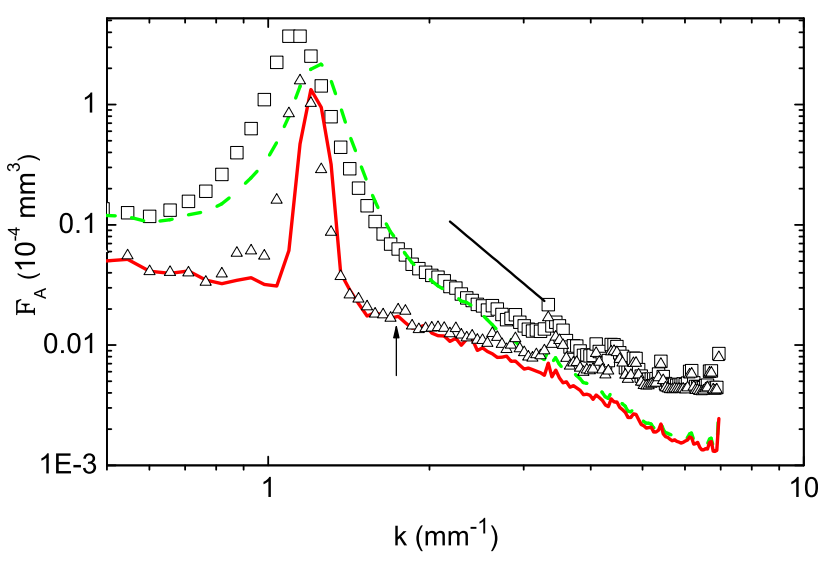

FIG. 4: (Color online) The temporal and angular averaged squared amplitude $\mathbf{F}_{A}$ of the Fourier spectrum of the interfacial states versus the radial averaged wave number $k$. Each curve is the average of 1000 images of the interfacial wave state. The lower liquid is the water sugar solution (symbols) or the $110 \mathrm{ppm}$ PEO solution (lines). The driving amplitudes are $a_{0}=31.7 \mathrm{~m} / \mathrm{s}^{2}$ (triangles and red line) and $a_{0}=60 \mathrm{~m} / \mathrm{s}^{2}$ (squares and green dashed line). The short black line indicates a $-15 / 4$ slope. The arrow indicates the position of the first higher order peak at $\sqrt{2} k_{0}$. depends on the shear rate. But shear thinning is more pronounced for concentrated solutions. The viscoelastic solutions used in the earlier Faraday Experiments [8] are characterized by a significant linear elastic contribution $\eta^{\prime \prime}(\omega)$ and shear thinning. Their interaction with the Faraday waves and the influence of the linear stability could be well explained in terms of a linear response theory.

The diluted low viscous polymer solution used in our experiments does not show an elastic contribution $\eta^{\prime}(\omega)$ that could be measured with a high resolution rheometer (MARS, Thermo Electron Germany, Karslruhe) and its shear viscosity measured at different constant shear rates $1<\dot{\gamma}<100$ differs only a few percent from the solvent viscosity. But diluted solutions of flexible polymers are known to possess a pronounced elongational viscosity $\eta_{e}$ 10]. Any flow can be separated into a rotational and an elongational part. This refers to the symmetric and the antisymmetric parts of the velocity gradient tensor $\mathbf{L}$, with components $L_{i j}=\nabla_{j} v_{i}$ ( $v_{i}$ are the velocity components of the flow). For Newtonian liquids $\eta_{e}$ is just given by a simple geometrical factor, the Trouton ratio with $\eta_{e}=3 \eta$. A pure rotation has no effect on the polymers but an elongation stretches them most efficiently at least when the Weisenberg number Wi, the product of elongational rate $\dot{\epsilon}$ and polymer relaxation time $\lambda$, exceeds a critical value $W i=\dot{\epsilon} \lambda>1 / 2$ [9, 13]. In simple shear flow the ratio between the symmetric and the antisymmetrical parts of the velocity gradient tensor is one. The rotational part leads to a tumbling movement of the polymers [12] and the stresses introduced by the elongational part are averaged over all orientations. In pure elongational flow the polymer stress can grow until the macroscopic elastic stress is orders of magnitude larger than the viscous part. Consequently, the elongational viscosities of our PEO solutions measured with a capillary break up extensional rheometer (CABER, Thermo Electron Germany, Karlsuhe) is in the order of several Pas, compared to $\eta_{e}=3 \times 7.2 m P a s$ for the solvent. Frequency dependent measurements of the complex elongational viscosity $\eta_{e}^{*}$ do not exist for our solutions, but for vanishing small amplitudes near threshold it should not differ from the complex shear viscosity $\eta^{*}$ in terms of a linear response theory.

We now can understand why the polymers do not affect the critical accelerations: It is a particularity of surface (or interfacial) waves that beside a small viscous sublayer near the interface the flow profile can be described by a potential and the flow is rotational free and purely elongational. The elastic part of the linear complex viscosity $\eta^{\prime \prime}$ of our solutions is too small and the dissipative part $\eta^{\prime}$ does not differ from the solvent viscosity. Only the contributions due to the pure elongational character of the flow might affect the polymers, but this occurs only for $W i>1 / 2$.

To estimate when the flow is strong enough to affect 


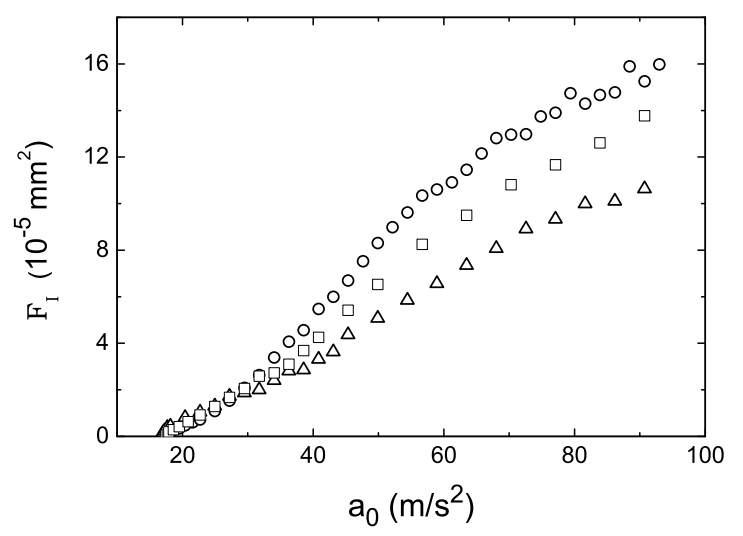

FIG. 5: The integrated intensity $\mathbf{F}_{I}$ of the temporal and angular averaged spatial Fourier spectrum (Fig. 4) of the interfacial states versus the driving acceleration. The lower liquid is the water sugar solution (circles), a 50 ppm PEO solution (squares) or the $110 \mathrm{ppm}$ PEO solution (triangles).

the polymers we look in a first approximation at only one spatial dimension. The velocity potential for a given wave number $k$ has the form

$$
\Phi=\omega / k A \sin (k x) \cosh (k z) \cos (\omega t)
$$

with $A$ the deformation amplitude of the wave and $z$ the direction of gravity. At the interface $z=0$ the flow is strongest and we can set $\cosh (k z)=1$. It follows for the amplitude $A_{L}$ of the velocity gradient tensor and thus the maximum elongational rate that occurs at the position of maximum peak elevation and at the interface $\dot{\epsilon}_{\max }=A_{L}=k \omega A$. Only at a driving strength of $a_{0}=40 \mathrm{~m} / \mathrm{s}^{2}$ the amplitude of the basic wave number is $A=1 \mathrm{~mm}$ and the elongational rate is $\dot{\epsilon}=2001 / \mathrm{s}$ and $W i=1.5$. At this driving strength the amplitude of the basic mode is strong enough to affect the polymers and we see differences in the amplitude of $F_{A}$ at $k_{0}$, compared to the pure sugar water solution. The integrated Intensity $F_{I}$ is dominated by the power of the fundamental mode and the effect can bee seen best in Fig. 5 However, already at low driving strength the wave number continuum at higher wave numbers adds to the velocity gradient tensor. The polymers get stretched by the high frequency fluctuations and their amplitude is reduced (Fig. 4). Apparently, these fluctuations are the reason for the disorder transition.

A comparison with drag reduction in fully developed turbulence is instructive. According to Landahl [14] the occurence of turbulent eddies in wall bounded systems like pipes is related to bursting motions, the so-called streaks, regions of flow with a high elongational component. The streaks occur near the wall and interact subtle with the turbulent eddies in which the energy is dissipated via a cascade to smaller lengths scales. The pronounced elongational viscosity is supposed to inhibit the streaks, yielding less eddies and less turbulent dissipation. Experimental findings support this picture and even a direct correlation of elongational viscosity and the ability of a polymer to perform to turbulent drag reduction has been found $[15]$.

In conclusion we have measured and described the effect of a small amount of a flexible polymer on the transition from an ordered to a weak turbulent state in parametrically driven interfacial waves. Without polymers we observe a rapid transition from a square to a disordered state with increasing driving strength. The addition of the polymers suppresses the high frequency fluctuations and stabilizes the ordered pattern. We find that the pronounced elongational viscosity is responsible for this effect and we suppose that further investigations on the effect of polymers on weak turbulent flow might help us to understand some of the key features of the scientific and technical important problem of turbulent drag reduction, too.

[1] B.A. Toms, Proc. Int. Cong. Rheol., II 135 (1949).

[2] J. L. Lumley , Ann. Rev. Fluid Mech. 1367 (1969). P. S. Virk, AIChE J. 25, 625 (1975). M. Tabor and de P. G. Gennes , Europhys. Lett. 2, 519 (1986). A. Gyr and H.W. Beersdorff, Drag recuction of turbulent flows by additives, (Kluwer, Dordrecht, 1995). J. Lumley and P. Blossey, Annu. Rev. Fluid Mech. 30, 311 (1998). V.S. L'vov, A. Pomyalov, I. Procaccia, and V. Tiberkevich, Phys. Rev. Lett. 92, 244503 (2004). D. Bonn, Yacine A., C. Wagner, S. Douady, and O. Cadot, J. Phys.: Condens. Matter 17, 1195 (2005).

[3] V. E. Zakharov and N. N Filonenko, J. Appl. Mech. Tech. Phys. 4, 506 (1967). V. E. Zakharov, V.S. L'vov, and G. Falkovich, Kolmogorov Spectra of turbulence I, Springer Verlag, Berlin. A. N. Pushkarev and V. E. Zakharov, Phys. Rev. Lett. 763320 (1996).

[4] A. V. Kityk, J. Embs, V. V. Mekhonoshin, and C. Wagner, Phys. Rev. E 72, 036209 (2005).

[5] W. B. Wright, R. Budakian, and S. J. Putterman, Phys. Rev. Lett. 76, 4528 (1996). E. Henry, P. Alstrøm, and M.T. Levinsen, Europhys. Lett. 52, 27 (2000). M. Yu. Brazhnikov, G.V. Kolmakov, A.A. Levchenko, and L.P. Mezhov-Deglin, Europhys. Lett. 58, 510 (2002).

[6] D. Binks and W. van de Water, Phys. Rev. Lett. 78, 4043 (1997).

[7] An extended presentation on the spatial and temporal spectra will be published elsewhere.

[8] F. Raynal, S. Kumar, and S. Fauve, Eur. Phys. J. B 9, 175 (1999). C. Wagner, H.W. Müller, and K. Knorr, Phys. Rev. Lett. 83, 308 (1999). O. Lioubashevski, Y. Hamiel, A. Agnon, Z. Reches, and J. Fineberg, Phys. Rev. Lett. 83, 3190 (1999).

[9] T. T. Perkins, D. E. Smith, and S. Chu, Science 276, 2016. D. E. Smith, H. P. Babcock, and S. Chu, Science 283, 1724 (1999). 
[10] Y. Amarouchene, D. Bonn, J. Meunier, and H. Kellay, Phys. Rev. Lett. 86, 3558 (2001).

[11] C. Wagner, Y. Amarouchene, D. Bonn, and J. Eggers, Phys. Rev. Lett. 95, 164504 (2005).

[12] C. M. Schroeder, R. E. Teixeira, E. S. G. Shaqfeh and S. Chu, Phys. Rev. Lett., 95, 18301 (2005).

[13] The relaxation time $\lambda$ for the PEO in water was determined by light scattering [10] and assuming a linear in- crease with the solvent viscosity we get $\lambda \approx 7$ ms. Higher values have been reported too [11], but they refer to large elongations.

[14] M.T. Landahl, Phys. Rev. Fluids 20, S55 (1977).

[15] C. Wagner, Y. Amarouchene, P. Doyle, and D. Bonn, Eur. Phys. Lett. 64, 823 (2003). 